\title{
The Impact of Blockchain in Smart Government Services
}

\author{
Entesar Ahmed ${ }^{1}$, Fatma Almarzooqi ${ }^{1}$, Marwa Bastaki ${ }^{1}$, Mira Bouka $^{1}$, and Dana Almidani ${ }^{1}$ \\ ${ }^{1}$ University of Wollongong Dubai
}

\begin{abstract}
Nowadays, blockchain technology is attracting massive attentions and trigger multiple projects. This research work aim at illustrating and analyzing the technology of blockchain and describe the different uses for it. Moreover, it discuss how technology impact the service sectors in industries by enhancing the quality of their services.
\end{abstract}

\section{Introduction}

Blockchain technology is basically a collection of data and transactions recorded and tracked through a network of distributed public ledgers and referred to as distributed ledger technology [1]. These ledgers are stored throughout the network, making it easy for users in terms of secure and auditable access. In different industries, blockchain technology is attracting massive attentions and trigger multiple projects [1-8]. The way we maintain administrative control has to change in digital world. Blockchain promise to solve this problem. In this research, the aim is to illustrate and analyze the technology of blockchain and describe the different uses for it. Moreover, describing how this technology could impact the service sectors in industries by enhancing the quality of their services. There are also some limitations for adopting this 
technology and some challenges that are still having to be figured all to avoid future risks. All these factors are summarized in this research. To describe briefly about this developed technology, it has been analyzed that it contains main different characteristics which will be defined specifically such as disintermediation, security, automation, immutability, trust, cost and traceability [7-16].

In our findings and recommendation for blockchain technology, researchers found that its based implementations still suffer from traditional challenges, such as lack of poor infrastructure and other technology issues. Also, a collaboration between industries and government to regulate the technology is one of important success factors for adopting. The benefits are huge if blockchain was developed in a right way, considering all the risks, as it helps in enhancing the transparency and reducing defects in service industries. In Brief, we have all heard that blockchain will revolutionize business, but it's going to take a lot longer than many people claim. The reason is that it's a foundational technology that will require a broad coordination, the level of regulatory, complexity, technological and social will be unprecedented. The truth is that the journey will take years for this new technology [1-26].

\section{Methodology}

This section presents the methodological approach considered in our study to show-case the concept of Blockchain technology, its evolvement, features/characteristics, limitations, as well as its impact on services in general and e-governments in particular. Accordingly, a structural research has been conducted where previous researches and studies have been investigated in different applications to learn blockchain concept from service design perspective and to identify 
the impact of this new technology in the public and private service sector. The aim was to recognize the role of the Blockchain technology in the services industry especially the E- services, its characteristics and impact on the smart services. As this technology is quite new, it was challenging to find reliable resources about this new concept and its applications. Our literature has been focused on limited keywords which were Block chain, blockchain, Smart services, Implementing blockchain, and the E- services. However, the study avoided the technical part of blockchain and excluded the topics related to the framework and structure of this technology. The research has excluded papers from conference that were not published. In this context, the study gathered forty articles from a well-known resources and databases such as Google scholar and Wollongong library which were released in the period from 2010 to date, and selected thirty different articles that were found more related to the subjects covered in this study. To be more specific, the study was divided into three parts. The first part was to understand the background of the block chain technology in terms of its concept, characteristics, advantages and applications. The second part was dedicated to understanding more about smart government services. The last part was to study the impact of the new technology of blockchain in the service industry. 
The articles give us a deeper understanding for each topic and the major topics have been selected as shown in below table.

\begin{tabular}{|c|c|c|}
\hline Number & Topic & Frequency \\
\hline $\mathbf{1}$ & $\begin{array}{c}\text { What is the Block chain } \\
\text { technology }\end{array}$ & 14 \\
\hline $\mathbf{2}$ & $\begin{array}{c}\text { Smart or E- services } \\
\text { The impact of block chain in } \\
\text { service industry } \\
\text { Excluded }\end{array}$ & 6 \\
\hline $\mathbf{4}$ & & 12 \\
\hline
\end{tabular}

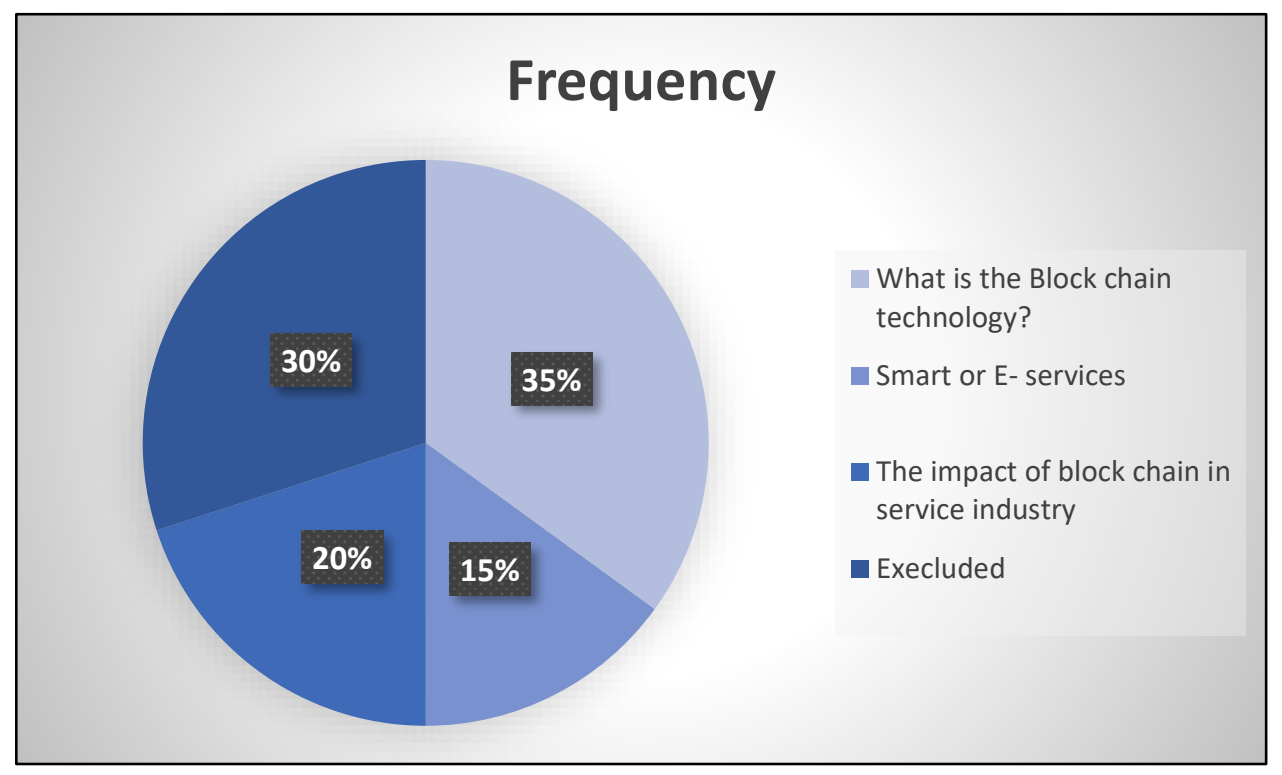




\section{Literature Review}

In this section, the block chain, the service system in e-government and smart government and the impact of the block chain in service system have been illustrated in order to be with the developments in the organizations which will lead to improve the quality and quantities of works inside the organizations and impact the companies with fast, organized and well-prepared products and services.

\section{Block Chain:}

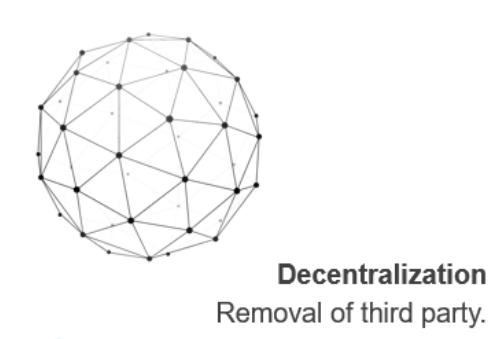

\section{BLOCKCHAIN}

BLOCKCHAIN CHARACHTRISTICS

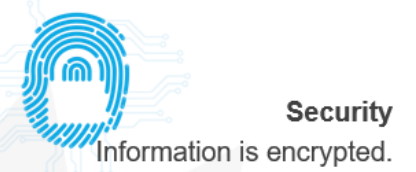

Information is encrypted.

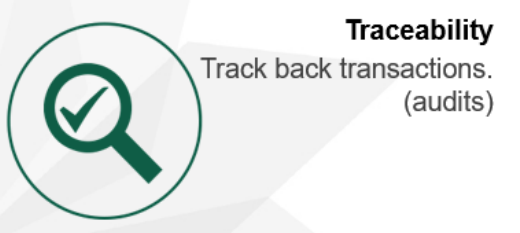

05

04

Persistency

Transactions can't be deleted or modified.

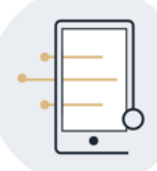

02

Anonymity

06

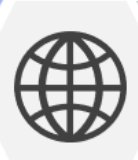

03

Anonymity

Blockchain doesn't disclose personal information.

Automation

E.g., Smart contract.

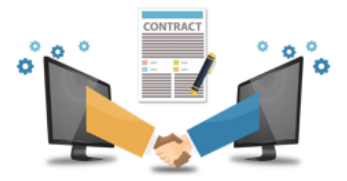

According to Conte De Leon, Stalick \& et al., (2017) Blockchain is a way of recording information using an encrypted ledger. The blockchain consists of nodes that can initiate and receive transactions. After a consensus approval of the transaction by all nodes, the transaction is then approved and added to the chain as a block. This new added 
information cannot be deleted, altered or manipulated by anyone. Moreover, it is one of the futuristic emerging technologies and it is based on decentralized database and it is a dynamic technology for problem solving creativity. It is based on peer to peer networking aiming to distribute data without any failure or mistakes [8-19]. The block chain is basically the idea of a completely stand-alone, autonomous, self-contained, self-validating application that does not depend on either immediate or eventual network communication is becoming nearly unthinkable [21].

Going through block chain Functionalities and Implications, it is designed of data sets which are composed of a chain of data packages (blocks) where a block comprises multiple transactions. The block chain is extended by each additional block where it represents a complete ledger of the transaction history [26].

According to Lu (2019) and Wang, Han \& et al., (2019) based on the application, block chain can be categorized into three categories; public chain, private chain and consortium chain. In the public chain which is also known as permissioned-less, the block chain is fully decentralized and anyone can participate in the process. In the private chain, which is also known as the permissioned, it is centralized and the access is controlled. Finally, the consortium chain which is also known as the hybrid, is partially distributed and the access is controlled by preselected members.

One of the advantages of block chain technology is that no single person has a control over the information. The entire data is available and visible to all parties, this mechanism secures the information and prevents any manipulation with it. Another advantage of this technology is traceability. Every piece of information along the supply 
chain can be traced back and investigated when needed. This will ensure product authenticity.

The characteristics of block chain have been defined as follow:

Decentralization: the standards of centralized transaction systems, the transactions need to be verified from central approved agency (central bank or others), which leads to cost and performance delay at the central servers. Differentiation to the centralized mode, third party is never again required in block chain [23-27].

$>$ Persistency: Transactions are hard to be deleted or restored once they are submitted in the block chain. Beside of that, it's easy to illustrate blocks that contain invalid transactions [18-26].

Anonymity: Every client can cooperate with the block chain with a produced address which doesn't shows the real ID of the client. (Zheng, Z \& et al., 2017)

> Auditability: Bitcoin block chain saves information about client balances formed on the Unspent Transaction Output (UTX-O) model (2); Transactions has to refer to previous unspent transactions. After the current transaction is saved into the block chain, the Unspent transaction changes from Unspent to spent which means that the transactions can be comfortably verified and tracked. (Crosby, M.\& et al., 2016)

$>$ Disintermediation - The peer-to-peer nature of the network means that a central authority is not needed. (Boucher \& et al.,2017) 
$>$ Security-The use of public key cryptography provides a high degree of confidence and security. The networked nature also provides a more resilient system with no single failure point. (Boucher \& et al.,2017)

$>$ Automation - Block chain is able to facilitate greater autonomous actions, such as smart contracts, increasing efficiency and accuracy. (Boucher \& et al.,2017)

$>$ Immutability- The data on the network is connected to preceding data meaning any attempt to alter data requires significant adjustments to multiple areas. (Boucher et al. ,2017)

$>$ Trust- With no third parties needing to be involved in a transaction, greater trust is possible and parties can deal directly with one another. (Boucher \& et al ,2017)

$>$ Costs - Costs can be cut due to the removal of third parties. (Boucher \& et al. ,2017)

The application fields for block chains seem to be manifold, especially in areas that have historically relied on third parties to establish a certain amount of trust. This disruptive innovation might change many existing business models, create new ones and might have severe impacts on entire industries. Therefore, research at the intersection of technology, markets and business models is certainly valuable. (Schiereck, 2017)

Blockchain can be favorable for some industries and sectors and unfavorable for some others. For example, in a regular business context, a smart contract in the supply chain doesn't rely on the intermediaries it is a direct deal between seller and buyer. After an agreement is reached between these two parties a contract is formalized and stored in a block chain structure. Accordingly, goods will be delivered, and money will be transferred in accordance to the contract's conditions. Each party in this network (the 
buyer and seller in this case) will be keeping a copy of this transaction ledger. (Queiroz, Telles, Bonilla, 2019).

In contrast, in sectors like the music sector the block chain has worsened the proper keeping of information. Music has always been shared in a non-standardized way. With the digital era, music has been shared and fractured across digital apps, websites, and media stores, etc. which made files to be surrounded by messy, imprecise information. (Baym, Swartz, Alarcon, 2019)

\section{Service in smart government:}

According to Guenduez, Singler \& et al., (2018) and Lv, li \& et al., (2018) the use of smart technologies has become an essential part in providing people with services in government sector. It is a new phenomenon that is becoming a main contributor for governments in providing their services to citizens. It is referred to as " Smart government". It can be defined as follow: it connects and integrates physical, public, digital and private environments to actively collaborate with citizens by better understanding their needs and provide services everywhere, anywhere, anytime. In other words, to benefit society in the best way possible.

According to Lv, li \& et al., (2018) smart governments are a result of experimenting with different types of technologies such as Internet of Things, Cloud Computing, Sensor Networks and Artificial Intelligence. However, the two most important types of information technology used in smart governments services are cloud computing and Internet of Things. Cloud computing is responsible for the storage, operation, decision-making, and command, while Internet of things is responsible for the function of information collection and automatic control. For example, in Switzerland, the police use a program called 
Precobs, which is a pre-crime observation system. It works as anti-burglary protection software. Precobs assumes that burglars strike several times within a short time if they are successful in a certain area. As a result, it is effective for the police to increase its presence and surveillance in that area (Guenduez, Singler \& et al., 2018).

Guenduez, Singler \& et al., (2018) and Mutiara, Yuniarti \& et al., (2018) both agree that the aim of smart government is to improve communication between government entities and citizens by giving them easy access to information, services and expertise. Another reason for implementing smart city is to improve the quality of life for citizens. Moreover, smart government is used to attract investors and create a sustainable urban environment. Smart government utilizes technology in order to provide easy and accessible services. Also, Smart governments utilizes technology to optimize public services. for example, in Indonesia, more than half of the citizens use Internet. Based on the increased number of internet users, government have focused on providing services through the internet.

In smart government concept, the SMART stands for social, mobile, analytics, radical openness and trust. While in M-government, according to OECD 2011, there are four delivery models which are: M-government to citizens, governments, business and to employees. A measurement metric for measuring the customer's satisfaction towards the M-government services was built as in four categories including: the value of money, quality of daily service, effective transaction and strategic data resources. Over all, the Egovernment best practices were classified and collected to be the international standards for governments and to develop from that more advantage in future. Examples including 
SMS notifications, ID sim, police applications and mobile parking fees payment (Ameen. A, 2018).

However, in order for smart government implementation in services to be successful, several factors must be taken into consideration. Firstly, technology management. Governments need to make sure that they have people with different skill sets to manage, plan, forecast and develop new applications for citizens. Secondly, cybersecurity. Ensuring all citizens' information are protected from hacking and from being misused. Thirdly, business analysis and modeling. Systematically identifying new requirements and finding solutions. it's related to process, service and structure changes. Finally, user experience is a key component of smart government success and focus on developing design thinking approach (Guenduez, Singler \& et al., 2018).

Digitization is so much important in every walk of life in the present world. The public sectors are also now modernizing their structures with many digital facilities and turning into smart governments, which help them closely study the relationship of public sector workers with their shareholders (Stephen, et al., 200). This e-government facilitates services to their natives through these electronic and Internet websites. Even though there had been many steps taken and there is promised success in the modernization of public sector, but there still is a very certain difference between the planning and their results (Stephen, et al., 200).

There are many barriers which are causing these obstacles in achieving the desired outcome. It is very important that how the organizations and their workers take these technologies, either they find them good or bad for their environment because their 
acceptance only will influence the success of the proposed technology (Stephen, et al., 200).

The economic exchange is firmly based on the creation of values. The orthodox method of creation of these values keeps the organization's output and price as the first priority (Schedle, et al., 2019). There are two main streams about this: Service science and Service dominant logic. This service system helps in growing the profit for the organization by giving importance to mutual benefits. The co-creation of value will help the organization in aiding the service science as well as other economic and social areas involved (Schedle, et al., 2019).

\section{Analysis \& Discussions}

\section{The impact of block chain in service system:}

Blockchain technologies have been increasingly promoted in governments data management. It is a cloud storage and application that supports decentralized data storage, archiving, analyzing, and manipulating. It also allows for the storage of data on different locations and systems which offers unparalleled data redundancy. Blockchain technology is true infrastructure as a service, storage as a service and data manipulation as a service. (Elisa et.al.,2018) which due to its algorithm complexity, schemes like Hadoop and Map Reduce can be used to access the data center. Blockchain aims to make optimal use of economy's resources where it pools in one space all transactions in the economy which would help in eliminating duplications and easier allocation of resources in an economical model. It also connects related past and future transactions where it keeps past records and creates updates and revisions for future records. 
Qi, et al., (2017), Ølnes, et al., (2017) and Navadkar, et al., (2018) all agree that governments from all over the world are conducting experiments to use blockchain to provide public services such as digital identity, marital status, e-voting, business licenses, passports, criminal records and even tax records. Also, several surveys and studies are being conducted on how to invest more in blockchain because, using blockchain in government services increases the quality by storing personal records, public records and individual certificated in the same platform. It also gives each individual a verifiable digital identity stored in the blockchain. As a result, the government will use this identity to provide public services which will lead to increasing effectiveness of government services and increasing speed of service delivery. It also aids in decreasing administrative bias by making sure everyone, equally, has access to government services (Navadkar, et al., 2018).

Qi, et al., (2017) and ØInes, et al., (2017) state that the application of blockchain in services is growing. For example, "smart contract" exists because blockchain technology facilitates the agreement between two or more parties on certain terms to do a specific job in exchange of something else. The main purpose of smart contract is automation which means that after the contract is approved, it will be self-executing, it doesn't need any contact between contractors. Also, the contract is able to arrange resources on its own behalf. This requires another technology used because of the blockchain which is "Smart Property".

"Smart Property" is another application of blockchain in service. Smart property is the ownership of properties by blockchain using smart contract. The properties are not limited to physical properties such as cars and houses, but it also includes intangible 
assets such as ideas, health data, rights and shares in a company. The ownership is represented by a private key on blockchain (Al-Shargie, Fares, 2016, Ølnes, et al., (2017)).

Nowadays, block chain is not only limited to the financial sectors, it has been applied to other industries such as food, healthcare, government and insurance. The technology helps those sectors that are particularly sensitive to products and their source of materials. For example, it can be used to trace back origin of food ingredients. Walmart performed an experiment that demonstrated that it only took 2.2 seconds to trace back mangos to their source, while using the old tracking method it took seven days (Wang, Han \& et al., 2019).

Additionally, E-commerce helps the government in their all projects and operations. Though it's e-commerce, but still the work of government on e-commerce is under human control which create many barriers. Whenever there's e commerce there's a threat of outside company and people barging in for the data illegally some of the individual are too careless or their mind can be manipulated easily to give off the data themselves or lose some very basic points of privacy helping other to break into the data. If the work of E-commerce is given upon the shoulders of individuals then it would have more chances for the information to leak and to overcome from those errors blockchain technology is proposed (Al-Shargie, Fares, 2018).

Blockchain is supposed to act in a way to minimize all the errors that are done by human strength. People now have huge expectation from blockchain as it creates a contact between different parties itself and helps them in collaborating. Blockchain system helps the organizations to examine the potential of the other parties. Blockchain provides 
trust to all the parties; it helps in decentralization and facilitates the formation and coordination of the systems (Al-Shargie, Fares, 2019)

For instance, The Swedish Mapping, Cadastre and Land Registration Authority at the beginning of 2017 launched a Blockchain project aiming to properly record and maintain the land title information and real estate transactions which should support faster and more transparent transactions, Eliminating duplications and selling properties to more than one person/entity, and greater security for system users.

Furthermore, the invention of bitcoin and the rise in its popularity helped blockchain to grow more. The popularity that gave light to the bitcoin also highlighted blockchain in every viewer's eyes. Blockchain is trustable as no party can own it, they all can only work upon it or work through it just like bitcoin, no one owns it. Since then blockchain has inspired many people and have grown too much in every platform (Al-Shargie, Fares, 2017).

One main concern of Blockchain has been evolving on government's data security. Eighty percent of e-government websites were exposed to the threat of scripting, service attacks and viruses targeting their connections such as US in 2015 they were hurt by losing over 4 million government employees' confidential information, also in Tanzania government, cyber-terrorists, technology spies, hackers and digital fraudsters incurred the government approximately 85 million dollar (Al-Shargie, Fares, 2019). This needs to be mitigated by using advanced encryption standards, such as the BB84 protocol. This advanced technology come to bring solutions to these incidents to ensure security and privacy and integrity of the e-government services through Blockchain technology where 
it provide a security for information exchange by establishing an environment of decentralization.

\section{Conclusion}

In this research, the discussion about the three main topics have been illustrated which are block chain technology, smart services in government and the impact of the new technology of block chain in the service industry, were some difficulties have been found when selecting the most reliable articles as they were limited since the topic is considered new to the service industry.

The block chain is basically the idea of a completely stand-alone, autonomous, selfcontained, self-validating application that does not depend on either immediate or eventual network communication is becoming nearly unthinkable. Block chain can be categorized into three categories; public chain, private chain and consortium chain. In the public chain which is also known as without permission, the block chain is fully decentralized and anyone can participate in the process. One of the advantages of block chain technology is that no single person has a control over the information. Characteristics of block chain have been defined as Decentralization, Persistency, Anonymity, Auditability, Disintermediation, Security, Automation, Immutability, Trust, Costs. Goods will be delivered, and money will be transferred in accordance to the contract's conditions. Each party in this network (the buyer and seller in this case) will be keeping a copy of this transaction ledger. In the music sector the block chain has worsened the proper keeping of information. Music has always been shared in a nonstandardized way. Nowadays, block chain is not only limited to the financial sectors, it has been applied to other industries such as food, healthcare, government and insurance. 
The use of smart technologies has become an essential part in providing people with services in government sector. It is a new phenomenon that is becoming a main contributor for governments in providing their services to citizens. It is referred to as "Smart government". Block chain has some shortcomings which needs to be fixed and addressed on timely basis so that it can become a part pf system and whole system can be dependent on it but unless or until these shortcomings won't get fixed it will be a huge mess to implement block chain system. Regulatory uncertainty is one big issue as if there is no proper regulation for block chain then there will be a potential threat of misuse of this technology which can result in harming industries, economies and world at large. Every new technology has hard time from users in terms of its authenticity and block chain has that as well but as due to its initial outburst in different forms it gives a sense of doubt among general people so it needs to gain trust of users first through its proper outline and systematic approach being transparent to users so that they can have trust levels back on blockchain. Due to its vague nature it's pretty unsalable business model right now and it's not a good sign for a technology as through proper systematic model approach and outline one can scale the business but in block chain all of the attributes are missing at this moment which needs to fixed so that it can become scalable and more users can take interest in it

\section{Recommendations}

As discussed, blockchain faces a lot of obstacles on applying it in smart government. So, in order to have an excellent implementation some recommendations have been determined as follow: 
* Measure the government readiness to apply new technology for example doing a clear analysis in each stage of implementing block chain

* Design strong fire wall before launching the system for external users

* Designed to accepts selective information from the customers to avoid duplication

* Design work shop for testing block chain technology before the launch

* Launch limited number of services to be able to react faster in case any incident occurs

* Access granted should follow special matrix of authority where segregation of duties is considered

* The quality of block chain should be measured by quality tools (servqual, ultimate questions or walk through audit).

* Sample random check on the function of the services provided every month to make sure the system is operating as it should be

* Create a research and development team where the services can be always updated and upgraded based on the customers need and demand

* Hiring a champion leader to lead the change along with critical mass

* Predicting the required time to implement the project within the time frame

* Provide required training in order to ensure everyone has the basic knowledge about this shift in service 


\section{References}

[1] Ameen. A, Al rajawy. I. "Proposing SMART-Government Model: Theoretical Framework". International Journal of Management and Human Science (IJMHS), Vol. 2, 2018, Iss. 2, pp 27-38.

[2] Al-Shargie, Fares, Masashi Kiguchi, Nasreen Badruddin, Sarat C. Dass, Ahmad Fadzil Mohammad Hani, and Tong Boon Tang. "Mental stress assessment using simultaneous measurement of EEG and fNIRS." Biomedical optics express 7, no. 10 (2016): 3882-3898.

[3] Al-Shargie, F. M., Tong Boon Tang, Nasreen Badruddin, and Masashi Kiguchi. "Mental stress quantification using EEG signals." In International Conference for Innovation in Biomedical Engineering and Life Sciences, pp. 15-19. Springer, Singapore, 2015.

[4] Al-Shargie, Fares, Tong Boon Tang, and Masashi Kiguchi. "Assessment of mental stress effects on prefrontal cortical activities using canonical correlation analysis: an fNIRS-EEG study." Biomedical optics express 8, no. 5 (2017): 2583-2598.

[5] Al-Shargie, Fares, Tong Boon Tang, and Masashi Kiguchi. "Stress assessment based on decision fusion of EEG and fNIRS signals." IEEE Access 5 (2017): 19889-19896.

[6] Al-Shargie, Fares, Tong Boon Tang, Nasreen Badruddin, and Masashi Kiguchi. "Towards multilevel mental stress assessment using SVM with ECOC: an EEG approach." Medical \& biological engineering \& computing 56, no. 1 (2018): 125-136.

[7] Al-shargie, Fares, Tong Boon Tang, and Masashi Kiguchi. "Mental stress grading based on fNIRS signals." In 2016 38th Annual International Conference of the IEEE Engineering in Medicine and Biology Society (EMBC), pp. 5140-5143. IEEE, 2016.

[8] Al-shargie, Fares, Tong Boon Tang, Nasreen Badruddin, and Masashi Kiguchi. "Simultaneous measurement of EEG-fNIRS in classifying and localizing brain activation to mental stress." In 2015 IEEE International Conference on Signal and Image Processing Applications (ICSIPA), pp. 282-286. IEEE, 2015.

[9] Al-Shargie, Fares. "Early Detection of Mental Stress Using Advanced Neuroimaging and Artificial Intelligence." arXiv preprint arXiv:1903.08511 (2019).

[10] Mohamed, Eltaf Abdalsalam, Mohd Zuki Yusoff, Ibrahim Khalil Adam, Elnazeer Ali Hamid, Fares Al-Shargie, and Muhammad Muzammel. "Enhancing EEG Signals in Brain Computer Interface Using Intrinsic Time-Scale Decomposition." In Journal of Physics: Conference Series, vol. 1123, no. 1, p. 012004. IOP Publishing, 2018.

[11] Al-shargie, Fares, Tong Boon Tang, Nasreen Badruddin, Sarat C. Dass, and Masashi Kiguchi. "Mental stress assessment based on feature level fusion of fNIRS and EEG signals." In 2016 6th International Conference on Intelligent and Advanced Systems (ICIAS), pp. 1-5. IEEE, 2016.

[12] Al-Shargie, Fares. "Multilevel Assessment of Mental Stress using SVM with ECOC: An EEG Approach." (2019).

[13] Al-Yoonus, Murthad, M. F. L. Abdullah, Mohammed Saeed Jawad, and Fares Al-Shargie. "Enhance quality control management for sensitive industrial products using 2D/3D image processing algorithms." In 2014 Electrical Power, Electronics, Communicatons, Control and Informatics Seminar (EECCIS), pp. 126-131. IEEE, 2014. 
[14] Al-Shargie, Fares. "Assessment of Mental Stress Using EEG and fNIRS Features." (2019).

[15] Jawad, Mohammed Saeed, Fares Al-Shargie, Murthad Al-Yoonus, and Zahriladha bin Zakaria. "Performance Analysis Comparative Study of Fingerprint Recognition Systems." Computers and Software (2014): 1154.

[16] Al-Shargie, Fares. "Quantification of Mental Stress using fNIRS Signals." (2019).

[17] Al-Shargie, Fares. "Fusion of fNIRS and EEG Signals: Mental Stress Study." (2019).

[18] Al-Shargie, Fares, Usman Tariq, Hasan Mir, Hamad Alawar, Fabio Babiloni, and Hasan AlNashash. "Vigilance decrement and enhancement techniques: a review." Brain sciences 9, no. 8 (2019): 178.

[19] Al-Shargie, Fares. "Assessment of Mental Stress among Undergraduate Students Using Novel Fusion Method on EEG and fNIRS Features." (2019).

[20] Al-Shargie, Fares. "Stress Detection and Reduction Based on Simultaneous Measurement of EEG and fNIRS Signals." (2019).

[21] Al-Shargie, Fares. "Vigilance Enhancement Using Traditional Methods: A Review." (2019).

[22] Al-Shargie, Fares. "Vigilance Enhancement Using Computerized Techniques." (2019).

[23] Al-Shargie, Fares, and CM GOH. "Cognitive Enhancement Techniques and Their Impact on Performance Improvements: A Review." (2019).

[24] Al-Shargie, Fares, U. Tariq, M. Alex, H. Mir, and H. Al-Nashash, "Emotion Recognition Based on Fusion of Local Cortical Activations and Dynamic Functional Networks Connectivity: An EEG Study," IEEE Access, vol. 7, pp. 143550-143562, 2019.

[25] Al-Shargie, Fares, Usman Tariq, Omnia Hassanin, Hasan Mir, Fabio Babiloni, and Hasan Al-Nashash. "Brain Connectivity Analysis Under Semantic Vigilance and Enhanced Mental States." Brain Sciences 9, no. 12 (2019): 363.

[26] Anjum, A., Sporny, M., \& Sill, A. (2017). Blockchain Standards for Compliance and Trust. Blockchain Standards for Compliance and Trust, 84-90.

[27] Baliga, A., 2016. The blockchain landscape. Persistent Systems. Baym, N., Swartz, L. and Alarcon, A., 2019. Sonic Publics| Convening 